Ann. Zootech., I978, 27 (3), 439-44I.

Note

\title{
Intềrêt des miçrobilles de verre comme marqueur de transit digestif chez le vison
}

\author{
J. ROUGEOT $\left({ }^{*}\right)$, Geneviève CHARLET-I,ERY (**) et A. ANDERSEN (***) \\ avec la collaboration technique de Michèle FIszLEwicz \\ (*) Laboratoire des Pelages, Toisons et Fourrures, \\ (**) Laboratoire de Physiologie de la Nutrition, \\ Centve national de Recherches zootechniques, I.N.R.A., \\ 78350 Jouy-en-Josas, France \\ (***) Faculté des Sciences de l'A griculture et de l'Alimentation, \\ Université Laval, Québec, P.Q. Canada GIK $7 P_{4}$
}

\begin{abstract}
Résumé
L'utilisation de microbilles de verre calibrées pour colonne à chromatographie, dans le but de mesurer le transit digestif chez le Vison, animal carnivore dont le tube digestif court ne possède aucun diverticule, semble intéressante. Leur récupération analytique par minéralisation KJELDAHL en est rapide et complète in vitro. In vivo, le comportement alimentaire du Vison gêne la mesure exacte de 1'ingéré qui est de ce fait fréquemment surestimé.
\end{abstract}

\section{Introduction}

I'étude de la vitesse du transit ou du temps de rétention des matériaux alimentaires dans le tube digestif fait appel à de nombreuses méthodes (LAPLACE, I972). Parmi les méthodes utilisées chez le Vison, les divers auteurs ont fait un choix identique : les matériaux colorés. SibBald et al. (1963) marquent un repas complet au carmin et contrôlent 1'apparition de la première émission colorée. Pour une étude quantitative, NESENI et PIATKows KI:(I958) utilisent des particules de paille colorées à la fuschine-diamant et, distribuant une boulette alimentaire incluant ces dernières, en mesurent les quantités retrouvées dans les émissions 
fécales et dans les diverses parties du tube digestif. Cette méthode a l'inconvénient d'être relativement longue.

L'utilisation de marqueurs chimiquement analysables, sesquioxyde de chrome ou célite, n'est pas possible vu le faible poids d'une émission fécale, de moins de I $g$ à $4 \mathrm{~g}$ maximum de matière sèche. Le marquage au ${ }^{141} \mathrm{Ce}$ (LAPLACE, I972), techniquement le meilleur, est onéreux et exige un appareillage de mesure de la radioactivité dont on ne dispose pas toujours.

La faible longueur du tube digestif du Vison - réduite à 4 fois celle du corps et sa simplicité anatomique (KAINER, I954; LAPLACE et ROUGEOT, I976), avec notamment un intestin de diamètre uniforme, sans cæcum et où l'on ne distingue ni morphologiquement, ni histologiquement l'intestin grêle du gros intestin, nous ont fait envisager la possibilité d'utiliser des microbilles de verre calibrées comme marqueur. En effet, les critiques dues à la densité d'un tel matériau ( $1,12 \mathrm{~g} / \mathrm{ml}$ ) valables pour des billes de diamètre important - environ $2 \mathrm{~mm} \mathrm{-.}$ (HOEIZEL, I930) le sont beaucoup moins lorsque la taille en est très réduite et plus proche des particules alimentaires.

\section{Matériel et méthodes}

Les microbilles utilisées sont des microbilles de verre calibrées à $5^{\circ} \mu \mathrm{m}$ pour colonne à chromatographie.

Pour le test in vitro, des quantités connues de microbilles sont mélangées à des fèces fraîches. Pour le test in vivo, 0,7 $\mathrm{g}$ de microbilles sont placées dans une boulette alimentaire d'environ Io $g$ composée par moitié de farine complète pour vison et de foie afin d'en augmenter l'appétence.

Cette boulette est offerte aux visons dans une coupelle : après $15 \mathrm{mn}$, le refus est recueilli pour analyse. Les collectes fécales sont effectuées dans des plateaux placés sous des cages de type classique, durant $24 \mathrm{~h}$, temps dépassant largement la durée des transits totaux : 5 à 7 h selon NESEnI et Piatikowski (I958).

Vingt-cinq journées de collecte ont été effectuées sur 4 visons. Par ailleurs, 26 animaux ont été abattus dans les 2 heures qui suivent l'ingestion et les contenus intestinaux ont été prélevés.

Chaque excrétion fécale ou échantillon digestif est séché aux rayons infrarouges durant $I_{5} \mathrm{~h}$ à $70^{\circ} \mathrm{C}$, pesé et mis en fioles $\mathrm{K}_{\text {JELDAHL }}$ de 300 à $500 \mathrm{ml}$ suivant la quantité; on ajoute, selon le cas, entre 30 et $50 \mathrm{ml} \mathrm{de} \mathrm{H}_{2} \mathrm{SO}_{4}$ concentré et environ $2 \mathrm{~g}$ de catalyseur laissant le moins possible de résidus ( $\mathrm{HgO}, \mathrm{K}_{2} \mathrm{SO}_{4}, \mathrm{CuSO}_{4}$ ). La minéralisation est poursuivie jusqu'à obtention d'une coloration verte stable, donc après solubilisation afin de permettre la filtration.

Cette filtration se fait sur une allonge entonnoir pour creusets filtrants placés sur une fiole à vide; l'orifice de cette allonge est obstrué par une bille de verre de $7 \mathrm{~mm}$ de diamètre retenant les billes et les particules supérieures à $25 \mu \mathrm{m}$ et laissant s'écouler le liquide et les matières minérales solides de très faible diamètre. Les billes sont récupérées sur un filtre en papier, séchées pendant $25 \mathrm{mn}$ dans une étuve à $100^{\circ} \mathrm{C}$, puis pesées par différence. Pour les excrétions les plus volumineuses, de grosses particules minérales peuvent rester avec les billes, mais il est très facile de les enlever avec une pince.

Si l'on désire effectuer simultanément le dosage de l'azote, la lyophilisation doit remplacer le séchage à l'étuve et la minération être prolongée le temps nécessaire. 


\section{Résultats et discussion}

La récupération analytique dans les mélanges fèces-microbilles est supérieure à $99 \mathrm{p}$ : I 00 .

In vivo, une mesure rigoureuse de l'ingéré permet une récupération totale ( 3 essais). Mais ce n'est pas toujours possible : cet animal a l'habitude de fouiller dans le récipient qui lui est présenté et projette un peu de nourriture hors de celui-ci. De plus, il transporte souvent de l'aliment dans son abreuvoir ou dans son nid. Quelques billes restent adhérentes au museau et aux moustaches de l'animal ou sur les mailles du grillage des cages, mais ne représentent que de très faibles quantités. L'ingéré est donc systématiquement surestimé.

Aussi, le taux de récupération apparaît toujours inférieur à roo. Il est respectivement de $9 \mathrm{I}, 3 \pm 0,95\left(^{*}\right)$ et de $90,0 \pm \mathrm{I}, 00$ pour les 20 collectes fécales et les 22 contenus digestifs des sujets qui ont peu gaspillé, de $87,8 \pm \mathrm{I}, 7$ et $86,9 \pm \mathbf{I}, 8$ pour la totalité des mesures effectuées ( 25 collectes fécales et 26 contenus digestifs).

I a simplicité analytique et 1'homogénéité des réponses montrent l'intérêt d'une telle méthode que les essais en cours préciseront.

Accepté pour publication en mai 1978 .

\section{Summary \\ Use of glass beads as digestive indicators in the mink}

The digestive tract of the mink is very simple. We therefore presumed that calibrated glass beads $(50 \mu \mathrm{m}$ for chromatography columns) could be used as external indicators for studying the rate of passage of digesta through this tract. Using the KJELDAHL method until total solubilization, the amount of glass beads was rapidly and accurately determined. When mixed in vitro with faeces, all beads were recovered. But, because the mink wastes feed, the intake of the glass beads was overestimated, as it exceeded the amount of beads recovered in the faeces (9r.33 \pm 0.95 p. roo) and digesta (90.o + r.oo p. I00). However, they were all recovered when the feed intake was fully controlled.

\section{Références bibliographiques}

HoElzel F., 1930. The rate of passage of inert materials through the digestive tract. $A \mathrm{~m} . J$. Physiol., 92, 466-497.

KAINER R. A., J954. The gross anatomy of the digestive system in the mink. Am.J. vet. Res., 15, 82-97.

IAPLACF, J. P., I972. Le transit digestif chez les monogastriques. I. Les techniques d'études. Ann. Zootech., 21, 83-105.

LAPLACE J. P., Rougeot J., I976. Mensurations viscérales chez le vison selon le sexe et le mode d'alimentation. Ann. Zootech., 25, 387-396.

Neseni R., Piatkowski B., I958. Durchgangszeiten des Futters beim Nerz. Arch. Tierernähr., 8, 296-307.

Sibbald I. R., Sinclair D. G., Evans E. V., Smith D. L. T,, ig62. The rate of passage of feed through the digestive tract of the mink. Can. J. Biochem. Physiol., 40, I39I-I 394. 